\title{
Couple of the Variational Iteration Method and Legendre Wavelets for Nonlinear Partial Differential Equations
}

\author{
Fukang Yin, ${ }^{1}$ Junqiang Song, ${ }^{1}$ Xiaoqun Cao, ${ }^{1}$ and Fengshun $\mathrm{Lu}^{2}$ \\ ${ }^{1}$ College of Computer, National University of Defense Technology, Changsha 410073, China \\ ${ }^{2}$ China Aerodynamics Research and Development Center, Mianyang, Sichuan 621000, China \\ Correspondence should be addressed to Fukang Yin; yinfukang@nudt.edu.cn
}

Received 17 October 2012; Revised 27 December 2012; Accepted 27 December 2012

Academic Editor: Francisco Chiclana

Copyright (c) 2013 Fukang Yin et al. This is an open access article distributed under the Creative Commons Attribution License, which permits unrestricted use, distribution, and reproduction in any medium, provided the original work is properly cited.

\begin{abstract}
This paper develops a modified variational iteration method coupled with the Legendre wavelets, which can be used for the efficient numerical solution of nonlinear partial differential equations (PDEs). The approximate solutions of PDEs are calculated in the form of a series whose components are computed by applying a recursive relation. Block pulse functions are used to calculate the Legendre wavelets coefficient matrices of the nonlinear terms. The main advantage of the new method is that it can avoid solving the nonlinear algebraic system and symbolic computation. Furthermore, the developed vector-matrix form makes it computationally efficient. The results show that the proposed method is very effective and easy to implement.
\end{abstract}

\section{Introduction}

Nonlinear phenomena are of fundamental importance in applied mathematics and physics and thus have attracted much attention. It is well known that most engineering problems are nonlinear, and it is very difficult to achieve the solution analytically or numerically. The analytical methods commonly used to solve them are very restricted, while the numerical techniques involving discretization of the variables on the other hand give rise to rounding off errors. Considerable attention has been paid to developing an efficient and fast convergent method. Recently, several approximate methods are introduced to find the numerical solutions of nonlinear PDEs, such as Adomian's decomposition method (ADM) [16], homotopy perturbation method (HPM) [7-12], homotopy analysis method (HAM) $[13,14]$, variational iteration method (VIM) [15-23], and wavelets method [24-29].

The variational iteration method (VIM) proposed by He [15-23] has been shown to be very efficient for handling a wide class of physical problems [16-18, 30-41]. If the exact solution of the nonlinear PDEs exists, the VIM gives rapidly convergent successive approximations; otherwise, a few approximations can be used for numerical purposes. In order to improve the efficiency of these algorithms, several modifications, such as variational iteration method using He's Polynomials [42-48] or using Adomian's Polynomials [49-54], have been developed and successfully applied to various engineering problems. However, since the variational iteration method provides the solution as a sequence of iterates, its successive iterations may be very complex, so that the resulting integrations in its iterative relation may be impossible to perform analytically.

In recent years, wavelets have found their way into many different fields of science and engineering. Various wavelets [24-29] have been used for studying problems with greater computational complexity and proved to be powerful tools to explore a new direction in solving differential equations. Unlike the variational iteration method that requires symbolic computations, the wavelets method converts the PDE into algebraic equations by the operational matrices, which can be solved by an iterative procedure. It is worthy to mention here that the method based on operational matrices of an orthogonal function for solving differential equations is computer oriented. The problem with this approach is that the algebraic equations may be singular and nonlinear.

Recently, some efficient modifications of ADM (using [55, 56]) and VIM or HAM [57] (using Legendre polynomials) are presented to approximate nonhomogeneous terms in 
nonlinear differential equations. Motivated and inspired by the ongoing research in these areas, we implement Legendre wavelets within the framework of VIM to facilitate the computational work of the method while still keeping the accuracy. The remainder of the paper is organized as follows. Section 2 introduces the VIM. In Section 3, we describe the basic formulation of Legendre wavelets and the operational matrix required for our subsequent development. In Section 4, we propose a new variational iteration method using Legendre wavelets (VIMLW). In order to demonstrate the validity and applicability of VIMLW, four examples are given in Section 5. Finally, a brief summary is presented.

\section{Variational Iteration Method}

This section introduces the basic ideas of variational iteration method (VIM). Here a description of the method [15-23] is given to handle the general nonlinear problem:

$$
L(u)+N(u)=g(t)
$$

where $L$ is a linear operator, $N$ is a nonlinear operator, and $g(t)$ is a known analytic function. According to He's VIM, we can construct a correction functional as follows:

$$
u_{n+1}(t)=u_{n}(t)+\int_{0}^{t} \lambda(\tau)\left\{L\left(u_{n}(\tau)\right)+N\left(\tilde{u}_{n}(\tau)\right)-g(\tau)\right\}
$$

where $\lambda$ is a general Lagrange multiplier which can be optimally identified via variational theory and $\widetilde{u}_{n}$ is a restricted variation which means $\delta \tilde{u}_{n}=0$. Therefore, the Lagrange multiplier $\lambda$ should be first determined via integration by parts. The successive approximation $u_{n}(t)(n \geq 0)$ of the solution $u(t)$ will be readily obtained by using the obtained Lagrange multiplier and any selective function $u_{0}$. The zeroth approximation $u_{0}$ may select any function that just meets, at least, the initial and boundary conditions. With $\lambda$ determined, several approximations $u_{n}(t), n \geq 0$, follow immediately. Consequently, the exact solution may be obtained as

$$
u(t)=\lim _{n \rightarrow \infty} u_{n}(t)
$$

The VIM depends on the proper selection of the initial approximation $u_{0}(t)$. Finally, we approximate the solution of the initial value problem (1) by the $n$ th-order term $u_{n}(t)$. It has been validated that VIM is capable of effectively, easily, and accurately solving a large class of nonlinear problems.

\section{Legendre Wavelets}

3.1. Legendre Wavelets. Legendre wavelets $\psi_{n m}(t)=\psi(k, \widehat{n}$, $m, t)$ have four arguments: $k$ is any positive integer, $\widehat{n}=$ $2 n-1\left(n=1,2,3, \ldots, 2^{k-1}\right), m$ is the order for Legendre polynomials, and $t$ is the normalized time. They are defined on the interval $[0,1)$ as follows:

$$
\begin{aligned}
& \psi_{n m}(t) \\
& = \begin{cases}\sqrt{m+1 / 2} 2^{(k / 2)} L_{m}\left(2^{k} t-\widehat{n}\right), & \text { for } \frac{\widehat{n}-1}{2^{k}} \leq t \leq \frac{\widehat{n}+1}{2^{k}} \\
0, & \text { otherwise, }\end{cases}
\end{aligned}
$$

where $m=0,1,2, \ldots, M-1, n=1,2, \ldots, 2^{k-1}$. The coefficient $\sqrt{m+1 / 2}$ is for orthonormality, the dilation parameter is $a=$ $2^{-k}$, and the translation parameter $b=\widehat{n} 2^{-k}$. Here, $L_{m}(t)$ are the well-known Legendre polynomials of order $m$ defined on the interval $[-1,1]$.

A function $f(t)$ defined over $[0,1)$ may be expanded by Legendre wavelet series as

$$
f(t)=\sum_{n=1}^{+\infty} \sum_{m=0}^{+\infty} c_{n m} \psi_{n m}(t)
$$

with

$$
c_{n m}=\left\langle f(t), \psi_{n m}(t)\right\rangle
$$

in $(6) ;\langle\cdot, \cdot\rangle$ denotes the inner product.

If the infinite series in (5) is truncated, then it can be written as

$$
f(t)=\sum_{n=1}^{2^{k-1}} \sum_{m=0}^{M-1} c_{n m} \psi_{n m}(t)=C^{T} \Psi(t)
$$

where $C$ and $\Psi(t)$ are $2^{k-1} M \times 1$ matrices given by

$$
\begin{aligned}
C(t)= & {\left[c_{10}, c_{11}, \ldots, c_{1 M-1}, c_{20}, \ldots, c_{2 M-1}, \ldots,\right.} \\
& \left.c_{2^{k-1} 0}, \ldots, c_{2^{k-1} M-1}\right]^{T} \\
\Psi(t)= & {\left[\psi_{10}(t), \psi_{11}(t), \ldots, \psi_{1 M-1}(t), \ldots,\right.} \\
& \left.\psi_{2^{k-1} 0}(t), \ldots, \psi_{2^{k-1} M-1}(t)\right]^{T}
\end{aligned}
$$

A two-dimensional function $f(x, t)$ defined over $[0,1) \times$ $[0,1)$ may be expanded by Legendre wavelet series as

$$
f(x, t)=\sum_{i=1}^{2^{k} M} \sum_{j=1}^{2^{k} M} c_{i j} \psi_{i}(x) \psi_{j}(t)=\Psi^{T}(x) C \Psi(t),
$$

with

$$
c_{i j}=\int_{0}^{1} f(x, t) \psi_{i}(x) d x \int_{0}^{1} f(x, t) \psi_{j}(t) d t .
$$

Equation (10) can be written into the discrete form (in matrix form) by

$$
f(x, t)=\Psi^{T}(x) C \Psi(t)
$$


where $C$ is a $2^{k-1} M \times 2^{k-1} M$ matrix given by

$$
C=\left[\begin{array}{cccc}
c_{0,0} & c_{0,1} & \cdots & c_{0,2^{k-1} M} \\
c_{1,0} & c_{1,1} & \cdots & c_{1,2^{k-1} M} \\
\vdots & \vdots & \ddots & \vdots \\
c_{2^{k-1} M, 0} & c_{2^{k-1} M, 1} & \cdots & c_{2^{k-1} M, 2^{k-1} M}
\end{array}\right]
$$

The integration and derivative operation matrices of the Legendre wavelets have been derived in $[58,59]$.

The integration of the vector $\Psi(t)$ defined in (9) can be obtained as

$$
\int_{0}^{t} \Psi(s) d s \cong P \Psi(t),
$$

where $P$ is a $2^{k-1} M \times 2^{k-1} M$ matrix given by [58].

The derivative of the vector $\Psi(t)$ can be expressed by

$$
\frac{d \Psi(t)}{d t}=D \Psi(t)
$$

where $D$ is the $2^{k-1} M \times 2^{k-1} M$ operational matrix of derivative given by [59].

The integration of $u(x, t)=\Psi^{T}(x) C \Psi(t)$ with respect to variable $t$ can be expressed as

$$
\begin{aligned}
\int_{0}^{t} u(x, \tau) d \tau & =\int_{0}^{t}\left(\Psi^{T}(x) C \Psi(\tau)\right) d \tau \\
& =\Psi^{T}(x) C\left(\int_{0}^{t} \Psi(\tau) d \tau\right)=\Psi^{T}(x) C P \Psi(t) .
\end{aligned}
$$

Similarly, the integration of $u(x, t)=\Psi^{T}(x) C \Psi(t)$ with respect to variable $x$ can be expressed as

$$
\begin{aligned}
\int_{0}^{x} u(\tau, t) d \tau & =\int_{0}^{x}\left(\Psi^{T}(\tau) C \Psi(t)\right) d \tau \\
& =\left(\int_{0}^{x} \Psi^{T}(\tau) d \tau\right) C \Psi(t)=\Psi^{T}(x) P^{T} C \Psi(t) .
\end{aligned}
$$

The derivative of $u(x, t)=\Psi^{T}(x) C \Psi(t)$ with respect to variable $t$ can be expressed as

$$
\begin{aligned}
\frac{\partial u(x, t)}{\partial t} & =\frac{\partial\left(\Psi^{T}(x) C \Psi(t)\right)}{\partial t}=\Psi^{T}(x) C \frac{\partial \Psi(t)}{\partial t} \\
& =\Psi^{T}(x) C D \Psi(t) .
\end{aligned}
$$

Similarly, the derivative of $u(x, t)=\Psi^{T}(x) C \Psi(t)$ with respect to variable $x$ can be expressed as

$$
\begin{aligned}
\frac{\partial u(x, t)}{\partial x} & =\frac{\partial\left(\Psi^{T}(x) C \Psi(t)\right)}{\partial x}=\frac{\partial \Psi^{T}(x)}{\partial x} C \Psi(t) \\
& =\Psi^{T}(x) D^{T} C \Psi(t) .
\end{aligned}
$$

3.2. Block Pulse Functions. The block pulse functions (BPFs) form a complete set of orthogonal functions that are defined on the interval $[0, b)$ by

$$
b_{i}(t)= \begin{cases}1, & \frac{i-1}{m} b \leq t<\frac{i}{m} b \\ 0, & \text { elsewhere }\end{cases}
$$

for $i=1,2, \ldots, m$. It is also known that for arbitrary absolutely integrable function $f(t)$ on $[0, b)$ can be expanded in block pulse functions:

$$
f(t) \simeq \xi^{T} B_{m}(t)
$$

in which

$$
\begin{gathered}
\xi^{T}=\left[f_{1}, f_{2}, \ldots, f_{m}\right], \\
B_{m}(t)=\left[b_{1}(t), b_{2}(t), \ldots, b_{m}(t)\right],
\end{gathered}
$$

where $f_{i}$ are the coefficients of the block pulse function given by

$$
f_{i}=\frac{m}{b} \int_{0}^{b} f(t) b_{i}(t) d t=\frac{m}{b} \int_{((i-1) / m) b}^{(i / m) b} f(t) b_{i}(t) d t .
$$

The elementary properties of BPFs are as follows.

(1) Disjointness: the BPFs are disjoined with each other in the interval $t \in[0, T)$ :

$$
b_{i}(t) b_{j}(t)=\delta_{i j} b_{i}(t)
$$

for $i, j=1,2, \ldots, m$.

(2) Orthogonality: the BPFs are orthogonal with each other in the interval $t \in[0, T)$ :

$$
\int_{0}^{T} b_{i}(t) b_{j}(t) d t=h \delta_{i j}
$$

for $i, j=1,2, \ldots, m$.

(3) Completeness: the BPFs set is complete when $m$ approaches infinity. This means that for every $f \in$ $L^{2}([0, T))$, when $m$ approaches to the infinity, Parseval's identity holds:

$$
\int_{0}^{T} f^{2}(t) d t=\sum_{i=1}^{\infty} f_{i}^{2}\left\|b_{i}(t)\right\|^{2}
$$

where

$$
f_{i}=\frac{1}{h} \int_{0}^{T} f(t) b_{i}(t) d t
$$

Definition 1. Let $A$ and $B$ be two matrices of $m \times m$, then $A \otimes$ $B=\left(a_{i j} \times b_{i j}\right)_{m m}$. 
Lemma 2. Assuming that $f(t)$ and $g(t)$ are two absolutely integrable functions, which can be expanded in block pulse function as $f(t)=F B(t)$, and $g(t)=G B(t)$ respectively, then one has

$$
f(t) g(t)=F B(t) B^{T}(t) G^{T}=H B(t),
$$

where $H=F \otimes G$.

Proof. According to the disjointness property of BPFS in (16), we have

$$
\begin{aligned}
F B(t) & B^{T}(t) G^{T} \\
= & {\left[\begin{array}{llll}
f_{11} g_{11} \phi_{1}(t) & f_{12} g_{12} \phi_{2}(t) & \cdots & f_{1 m} g_{1 m} \phi_{2}(t)
\end{array}\right] } \\
= & H B(t) .
\end{aligned}
$$

Lemma 3. Let $f(x, t)$ and $g(x, t)$ be two absolutely integrable functions, which can be expanded in block pulse function as $f(x, t)=B^{T}(x) F B(t)$ and $g(x, t)=B^{T}(x) G B(t)$, respectively, one has

$$
f(x, t) g(x, t)=B^{T}(x) H B(t),
$$

where $H=F \otimes G$.

3.3. Nonlinear Term Approximation. The Legendre wavelets can be expanded into $m$-set of block pulse functions as

$$
\Psi(t)=\Phi_{m \times m} B_{m}(t) .
$$

Taking the collocation points as follow,

$$
t_{i}=\frac{i-1 / 2}{2^{k-1} M}, \quad i=1,2, \ldots, 2^{k-1} M .
$$

The $m$-square Legendre matrix $\Phi_{m \times m}$ is defined as

$$
\Phi_{m \times m} \triangleq\left[\begin{array}{llll}
\Psi\left(t_{1}\right) & \Psi\left(t_{2}\right) & \cdots & \Psi\left(t_{2^{k-1} M}\right)
\end{array}\right] .
$$

The operational matrix of product of Legendre wavelets can be obtained by using the properties of BPFs. Let $f(x, t)$ and $g(x, t)$ be two absolutely integrable functions, which can be expanded in Legendre wavelets as $f(x, t)=\Psi^{T}(x) F \Psi(t)$ and $g(x, t)=\Psi^{T}(x) G \Psi(t)$, respectively.

From (31), we have

$$
\begin{aligned}
& f(x, t)=\Psi^{T}(x) F \Psi(t)=B^{T}(x) \Phi_{m m}^{T} F \Phi_{m m} B(t), \\
& g(x, t)=\Psi^{T}(x) G \Psi(t)=B^{T}(x) \Phi_{m m}^{T} G \Phi_{m m} B(t),
\end{aligned}
$$

and let $F_{b}=\Phi_{m m}^{T} F \Phi_{m m}, G_{b}=\Phi_{m m}^{T} G \Phi_{m m}, H_{b}=F_{b} \otimes G_{b}$.

By employing Lemma 3 , we get

$$
\begin{aligned}
f(x, t) g(x, t)= & B^{T}(x) H_{b} B(t) \\
= & B^{T}(x) \Phi_{m m}^{T} \operatorname{inv}\left(\Phi_{m m}^{T}\right) H_{b} \operatorname{inv}\left(\Phi_{m m}\right) \\
& \times \Phi_{m m} B(t) \\
= & \Psi^{T}(x) H \Psi(t),
\end{aligned}
$$

where $H=\operatorname{inv}\left(\Phi_{m m}^{T}\right) H_{b} \operatorname{inv}\left(\Phi_{m m}\right)$.

\section{Variational Iteration Method Using Legendre Wavelets}

In this section, we present a new modification of variational iteration method using Legendre wavelets (called VIMLW). This algorithm can be implemented for solving nonlinear PDEs effectively.

To deduce the basic relations of our proposed algorithm, consider the following forms of initial value problems:

$$
L[u(x, t)]+N[u(x, t)]=g(x, t), \quad x \in[0,1], t>0,
$$

where $L$ and $N$ are linear operator and nonlinear operator, respectively, and $g(x, t)$ is a known analytic function, subject to the initial condition $u(x, 0)$. It should be noted here that $L[u(x, t)]$ contains the term $\partial^{m} u / \partial t^{m}$, where $m$ is a positive integer.

According to the traditional VIM, we can construct the correction functional for (36) as

$$
\begin{gathered}
u_{k+1}(x, t)=u_{k}(x, t)+\int_{0}^{t} \lambda\left[L\left(u_{k}(x, \tau)\right)+N\left(u_{k}(x, \tau)\right)\right. \\
-g(x, \tau)] d \tau .
\end{gathered}
$$

The Lagrange multiplier of (37) is

$$
\lambda(t, \tau)=-\frac{(t-\tau)^{m-1}}{(m-1) !}=\frac{(-1)^{m}(\tau-t)^{m-1}}{(m-1) !} .
$$

In order to improve the performance of VIM, we introduce Legendre wavelets to approximate $u_{k}(x, t)$ and the nonhomogeneous term $g(x, t)$ as

$$
u_{k}(x, t)=\Psi^{T}(x) C_{k} \Psi(t), \quad g(x, t)=\Psi^{T}(x) G \Psi(t) .
$$

Now for the nonlinear part, by nonlinear term approximation described in Section 3.3, we have

$$
N\left[u_{k}(x, t)\right]=\Psi^{T}(x) N_{k} \Psi(t),
$$

where $N$ is matrix of order $2^{k-1} M \times 2^{k^{\prime}-1} M^{\prime}$.

For the linear part, we have

$$
L\left[u_{k}(x, t)\right]=\Psi^{T}(x) L_{k} \Psi(t),
$$

where $L$ is a matrix of order $2^{k-1} M \times 2^{k^{\prime}-1} M^{\prime}$.

Then the iteration formula (37) can be constructed as

$$
\begin{aligned}
\Psi^{T}(x) C_{k+1} \Psi(t)= & \Psi^{T}(x) C_{k} \Psi(t) \\
& +\int_{0}^{t} \lambda \Psi^{T}(x)\left[L_{k}+N_{k}-G\right] \Psi(\tau) d \tau .
\end{aligned}
$$

If $\lambda$ is constant, we have

$$
C_{k+1}=C_{k}+\lambda\left[L_{k}+N_{k}-G\right] P .
$$


TABLE 1: Numerical values when $t=0.25,0.50,0.75$, and 1.0 for (52).

\begin{tabular}{|c|c|c|c|c|c|c|c|c|}
\hline$t$ & \multicolumn{4}{|c|}{0.25} & \multicolumn{4}{|c|}{0.50} \\
\hline$x$ & 0.25 & 0.50 & 0.75 & 1.00 & 0.25 & 0.50 & 0.75 & 1.00 \\
\hline VIM & 0.20002 & 0.40004 & 0.60006 & 0.80007 & 0.16702 & 0.33405 & 0.50107 & 0.66810 \\
\hline VIMLW & 0.20002 & 0.40004 & 0.60006 & 0.80006 & 0.16702 & 0.33405 & 0.50107 & 0.66809 \\
\hline Exact & 0.20000 & 0.40000 & 0.60000 & 0.80000 & 0.16667 & 0.33333 & 0.50000 & 0.66667 \\
\hline$t$ & \multicolumn{4}{|c|}{0.75} & \multicolumn{4}{|c|}{1.00} \\
\hline$x$ & 0.25 & 0.50 & 0.75 & 1.00 & 0.25 & 0.50 & 0.75 & 1.00 \\
\hline VIM & 0.14461 & 0.28921 & 0.43382 & 0.57842 & 0.12989 & 0.25977 & 0.38966 & 0.51955 \\
\hline VIMLW & 0.14460 & 0.28921 & 0.43382 & 0.57841 & 0.12989 & 0.25977 & 0.38966 & 0.51953 \\
\hline Exact & 0.14286 & 0.28571 & 0.42857 & 0.57143 & 0.12500 & 0.25000 & 0.37500 & 0.50000 \\
\hline
\end{tabular}

When $\lambda$ is a function of $\tau$, the Legendre wavelets are used to approximate $\lambda(\tau)$ as

$$
\lambda(t, \tau)=\Psi^{T}(t) S \Psi(\tau) .
$$

Substituting (44) into (42), we have

$$
\begin{aligned}
\Psi^{T}(x) & C_{k+1} \Psi(t) \\
= & \Psi^{T}(x) C_{k} \Psi(t) \\
& +\int_{0}^{t} \Psi^{T}(x)\left[L_{k}+N_{k}-G\right] \Psi(\tau) \Psi^{T}(\tau) S^{T} \Psi(t) d \tau .
\end{aligned}
$$

Since

$$
\Psi(\tau)=\left[\begin{array}{cccc}
\phi_{11} & \phi_{12} & \cdots & \phi_{1 m} \\
\phi_{21} & \phi_{22} & \cdots & \phi_{2 m} \\
\vdots & \vdots & \ddots & \vdots \\
\phi_{m 1} & \phi_{m 2} & \cdots & \phi_{m m}
\end{array}\right]\left[\begin{array}{c}
B_{1}(\tau) \\
B_{2}(\tau) \\
\vdots \\
B_{m}(\tau)
\end{array}\right]=\left[\begin{array}{c}
\sum_{i=1}^{m} \phi_{1 i} B_{i}(\tau) \\
\sum_{i=1}^{m} \phi_{2 i} B_{i}(\tau) \\
\vdots \\
\sum_{i=1}^{m} \phi_{m i} B_{i}(\tau)
\end{array}\right],
$$

we get

$$
\begin{aligned}
& \Psi(\tau) \Psi^{T}(\tau)=\left[\begin{array}{c}
\sum_{i=1}^{m} \phi_{1 i} B_{i}(t) \\
\sum_{i=1}^{m} \phi_{2 i} B_{i}(t) \\
\vdots \\
\sum_{i=1}^{m} \phi_{m i} B_{i}(t)
\end{array}\right] \\
& \times\left[\sum_{i=1}^{m} \phi_{1 i} B_{i}(t) \sum_{i=1}^{m} \phi_{2 i} B_{i}(t) \cdots \sum_{i=1}^{m} \phi_{m i} B_{i}(t)\right] \\
& =\sum_{j=1}^{m}\left(\sum_{i=1}^{m} \phi_{j i} B_{i}(t)\right)^{2} \text {. }
\end{aligned}
$$

where

According to the property of block pulse functions, we obtain

$$
\begin{aligned}
\Psi(\tau) & \Psi^{T}(\tau) \\
& =\sum_{j=1}^{m} \sum_{i=1}^{m} \phi_{j i}^{2} B_{i}(t) \\
& =\left[\begin{array}{cccc}
\phi_{11}^{2} & \phi_{12}^{2} & \cdots & \phi_{1 m}^{2} \\
\phi_{21}^{2} & \phi_{22}^{2} & \cdots & \phi_{2 m}^{2} \\
\vdots & \vdots & \ddots & \vdots \\
\phi_{m 1}^{2} & \phi_{m 2}^{2} & \cdots & \phi_{m m}^{2}
\end{array}\right]\left[\begin{array}{c}
B_{1}(t) \\
B_{2}(t) \\
\vdots \\
B_{m}(t)
\end{array}\right]\left[\begin{array}{llll}
1 & 1 & \cdots & 1
\end{array}\right]
\end{aligned}
$$

$$
\begin{aligned}
& {\left[\begin{array}{cccc}
\phi_{11}^{2} & \phi_{12}^{2} & \cdots & \phi_{1 m}^{2} \\
\phi_{21}^{2} & \phi_{22}^{2} & \cdots & \phi_{2 m}^{2} \\
\vdots & \vdots & \ddots & \vdots \\
\phi_{m 1}^{2} & \phi_{m 2}^{2} & \cdots & \phi_{m m}^{2}
\end{array}\right] \operatorname{inv}\left(\Phi_{m \times m}\right) \Psi(t)\left[\begin{array}{llll}
1 & 1 & \cdots & 1
\end{array}\right] } \\
= & H \Psi(t)\left[\begin{array}{llll}
1 & 1 & \cdots & 1
\end{array}\right],
\end{aligned}
$$

$$
H=\left[\begin{array}{cccc}
\phi_{11}^{2} & \phi_{12}^{2} & \cdots & \phi_{1 m}^{2} \\
\phi_{21}^{2} & \phi_{22}^{2} & \cdots & \phi_{2 m}^{2} \\
\vdots & \vdots & \ddots & \vdots \\
\phi_{m 1}^{2} & \phi_{m 2}^{2} & \cdots & \phi_{m m}^{2}
\end{array}\right] \operatorname{inv}\left(\Phi_{m \times m}\right)
$$

Substituting (48) into (45), we have

$$
\begin{aligned}
& \Psi^{T}(x) C_{k+1} \Psi(t) \\
& =\Psi^{T}(x) C_{k} \Psi(t)+\int_{0}^{t} \Psi^{T}(x)\left[L_{k}+N_{k}-G\right] \\
& \times H \Psi(\tau)\left[\begin{array}{llll}
1 & 1 & \cdots & 1
\end{array}\right] S^{T} \Psi(t) d \tau
\end{aligned}
$$


TABLE 2: Numerical values when $t=0.25,0.50,0.75$, and 1.0 for (54).

\begin{tabular}{|c|c|c|c|c|c|c|c|c|}
\hline$t$ & \multicolumn{4}{|c|}{0.25} & \multicolumn{4}{|c|}{0.50} \\
\hline$x$ & 0.25 & 0.50 & 0.75 & 1.00 & 0.25 & 0.50 & 0.75 & 1.00 \\
\hline VIM & 0.05001 & 0.20002 & 0.45004 & 0.80007 & 0.04176 & 0.16702 & 0.37581 & 0.66810 \\
\hline VIMLW & 0.05000 & 0.20002 & 0.45004 & 0.80021 & 0.04168 & 0.16699 & 0.37572 & 0.66831 \\
\hline Exact & 0.05000 & 0.20000 & 0.45000 & 0.80000 & 0.04167 & 0.16667 & 0.37500 & 0.66667 \\
\hline$t$ & \multicolumn{4}{|c|}{0.75} & \multicolumn{4}{|c|}{1.00} \\
\hline$x$ & 0.25 & 0.50 & 0.75 & 1.00 & 0.25 & 0.50 & 0.75 & 1.00 \\
\hline VIM & 0.03615 & 0.14461 & 0.32536 & 0.57842 & 0.03247 & 0.12989 & 0.29225 & 0.51955 \\
\hline VIMLW & 0.03577 & 0.14443 & 0.32491 & 0.57751 & 0.03128 & 0.12932 & 0.29083 & 0.51421 \\
\hline Exact & 0.03571 & 0.14286 & 0.32143 & 0.57143 & 0.03125 & 0.12500 & 0.28125 & 0.50000 \\
\hline
\end{tabular}
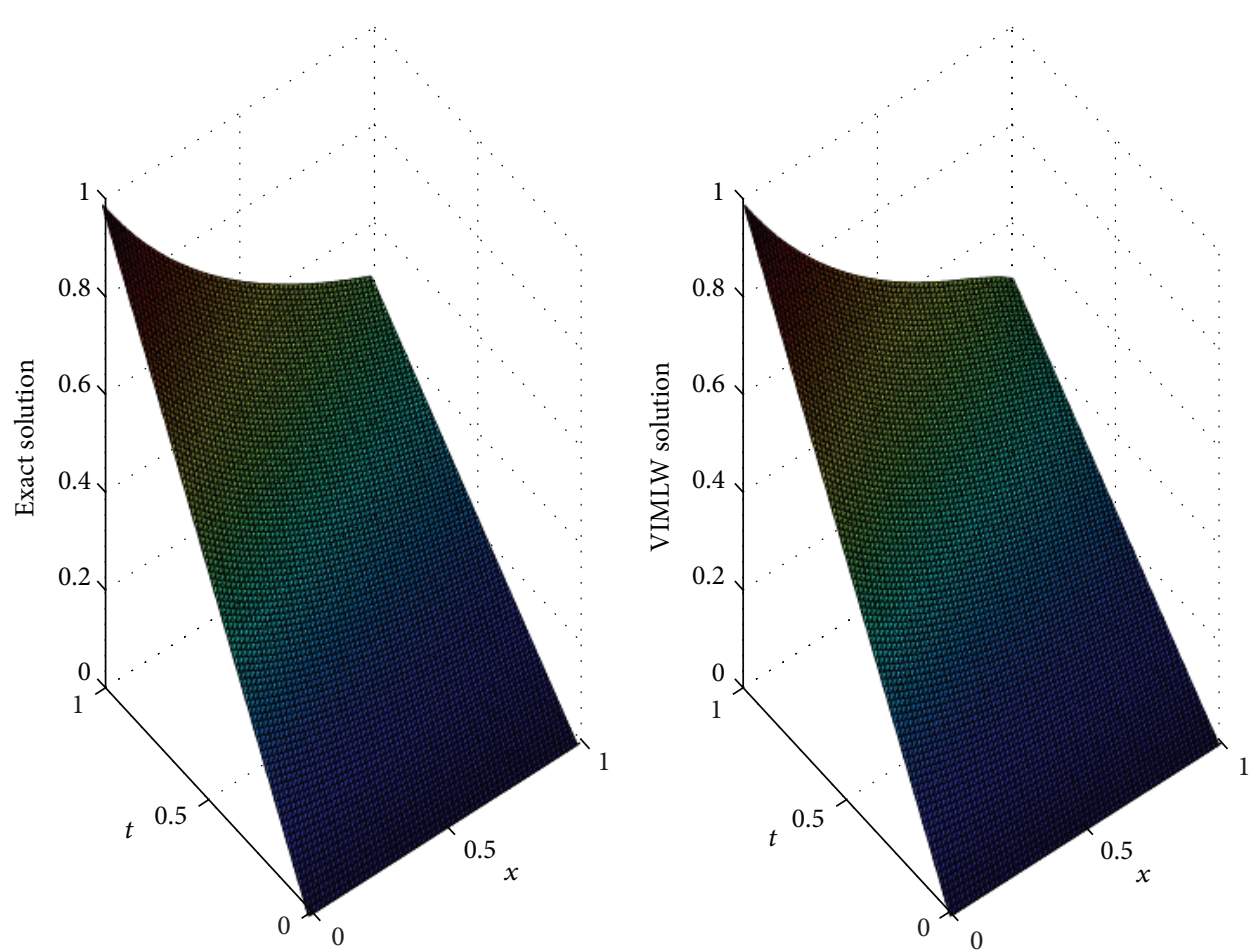

FIGURE 1: Exact solution and VIMLW approximate solution of Example 4.

$$
\begin{aligned}
= & \Psi^{T}(x) C_{k} \Psi(t)+\Psi^{T}(x)\left[L_{k}+N_{k}-G\right] \\
& \times H P \Psi(t)\left[\begin{array}{llll}
1 & 1 & \cdots & 1
\end{array}\right] S^{T} \Psi(t) \\
= & \Psi^{T}(x) C_{k} \Psi(t)+\Psi^{T}(x)\left[L_{k}+N_{k}-G\right] \\
& \times H P \Psi(t) \Psi^{T}(t) S\left[\begin{array}{llll}
1 & 1 & \cdots & 1
\end{array}\right]^{T} \\
= & \Psi^{T}(x) C_{k} \Psi(t)+\Psi^{T}(x)\left[L_{k}+N_{k}-G\right] \\
& \times H P H \Psi(t) s,
\end{aligned}
$$

where $s=\left[\begin{array}{llll}1 & 1 & \cdots & 1\end{array}\right] S\left[\begin{array}{llll}1 & 1 & \cdots & 1\end{array}\right]^{T}$.

Finally, we get the iteration formula as follows:

$$
C_{k+1}=C_{k}+\left[L_{k}+N_{k}-G\right] H P H\left[\begin{array}{llll}
1 & 1 & \cdots & 1
\end{array}\right] S\left[\begin{array}{llll}
1 & 1 & \cdots & 1
\end{array}\right]^{T} \text {. }
$$

\section{Numerical Examples}

To demonstrate the effectiveness and good accuracy of the VIMLW, four different examples will be examined.

Example 4. Consider the regularized long-wave (RLW) equation [39]:

$$
u_{t}-u_{x x t}+\left(\frac{u^{2}}{2}\right)_{x}=0, \quad-\infty<x<\infty, t>0
$$

with the initial condition $u(x, 0)=x$ and the exact solution is $u(x, t)=(x /(1+t))$. 

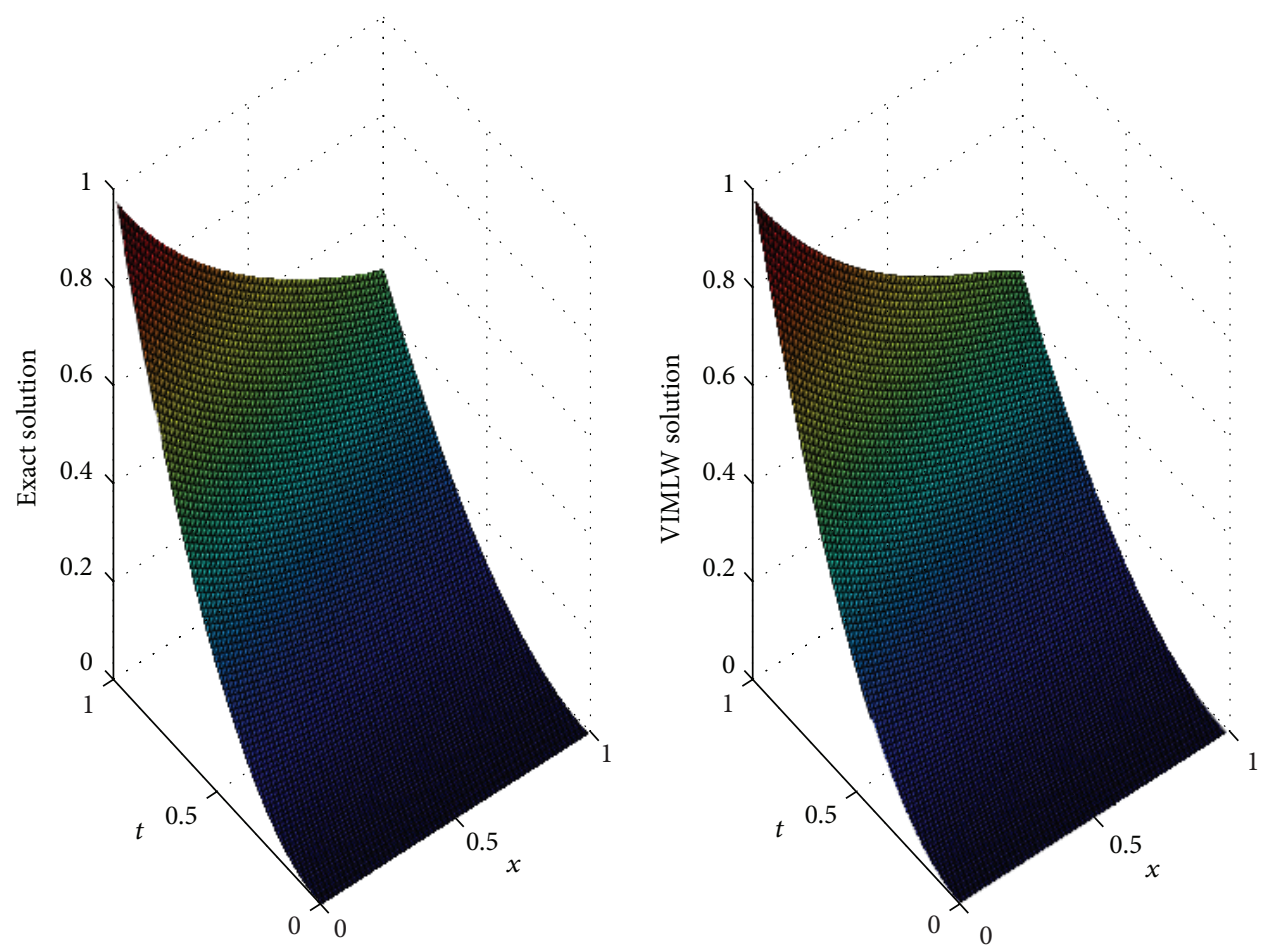

Figure 2: Exact solution and VIMLW approximate solution of Example 5.
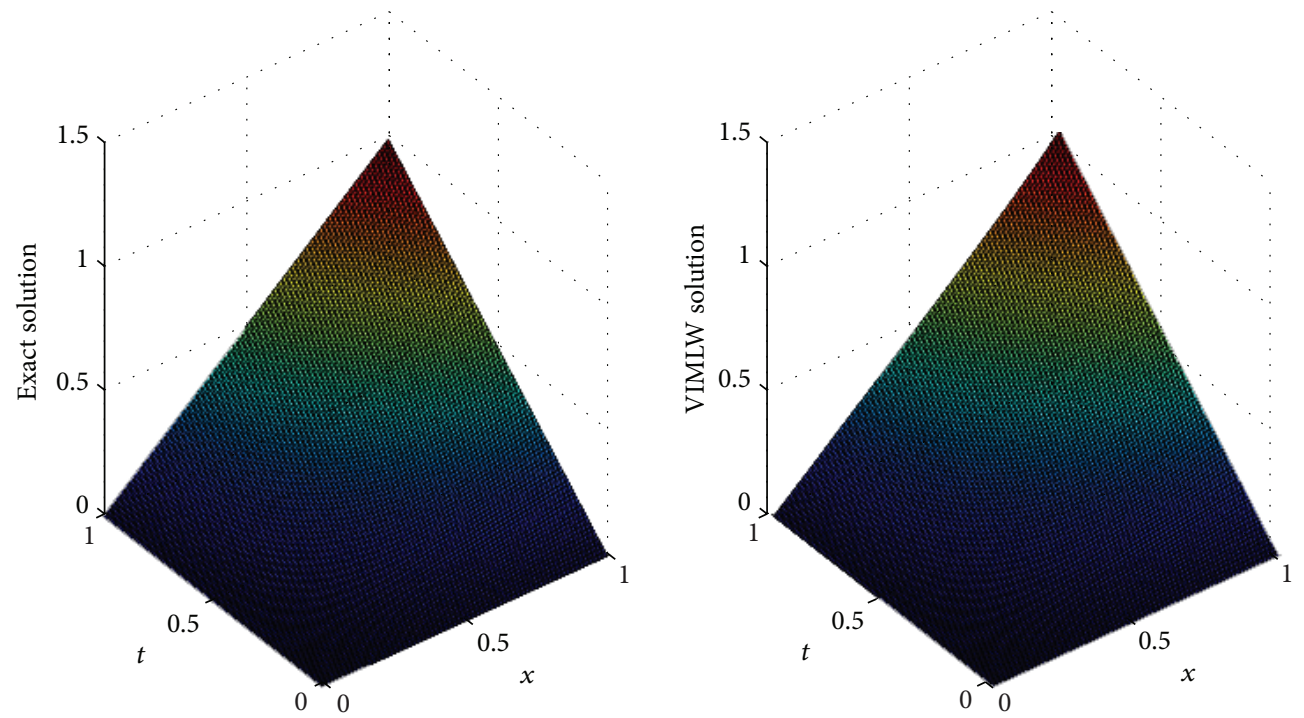

FIgURE 3: Exact solution and VIMLW approximate solution of Example 6.

By assuming $u_{k}(x, t)=\Psi^{T}(x) C_{k} \Psi(t)$ and from (52), we have

$$
\begin{gathered}
L\left[u_{k}\right]=\frac{\partial u_{k}}{\partial t}-\frac{\partial^{3} u_{k}}{\partial x^{2} \partial t}=\Psi^{T}(x) L_{k} \Psi(t), \\
N\left[u_{k}\right]=u_{k} \frac{\partial u_{k}}{\partial x}=\Psi^{T}(x) N_{k} \Psi(t),
\end{gathered}
$$

where $L_{k}=C_{k} D-\left(D^{T}\right)^{2} C_{k} D, N_{k}=\left(D^{T} C_{k}\right) \otimes C_{k}$.
We utilize the methods presented in this paper to solve (52) with $M=16$ and $k=1$. Table 1 shows the approximate solutions for (52) obtained for different points using the variational iteration and VIMLW method. Figure 1 presents the Exact solution and VIMLW approximate solution of Example 4. Note that only the fifth-order term of their solutions is used in evaluating the approximate solutions for Example 4. We can see that the approximate solution obtained with VIMLW gives almost the same results as that 

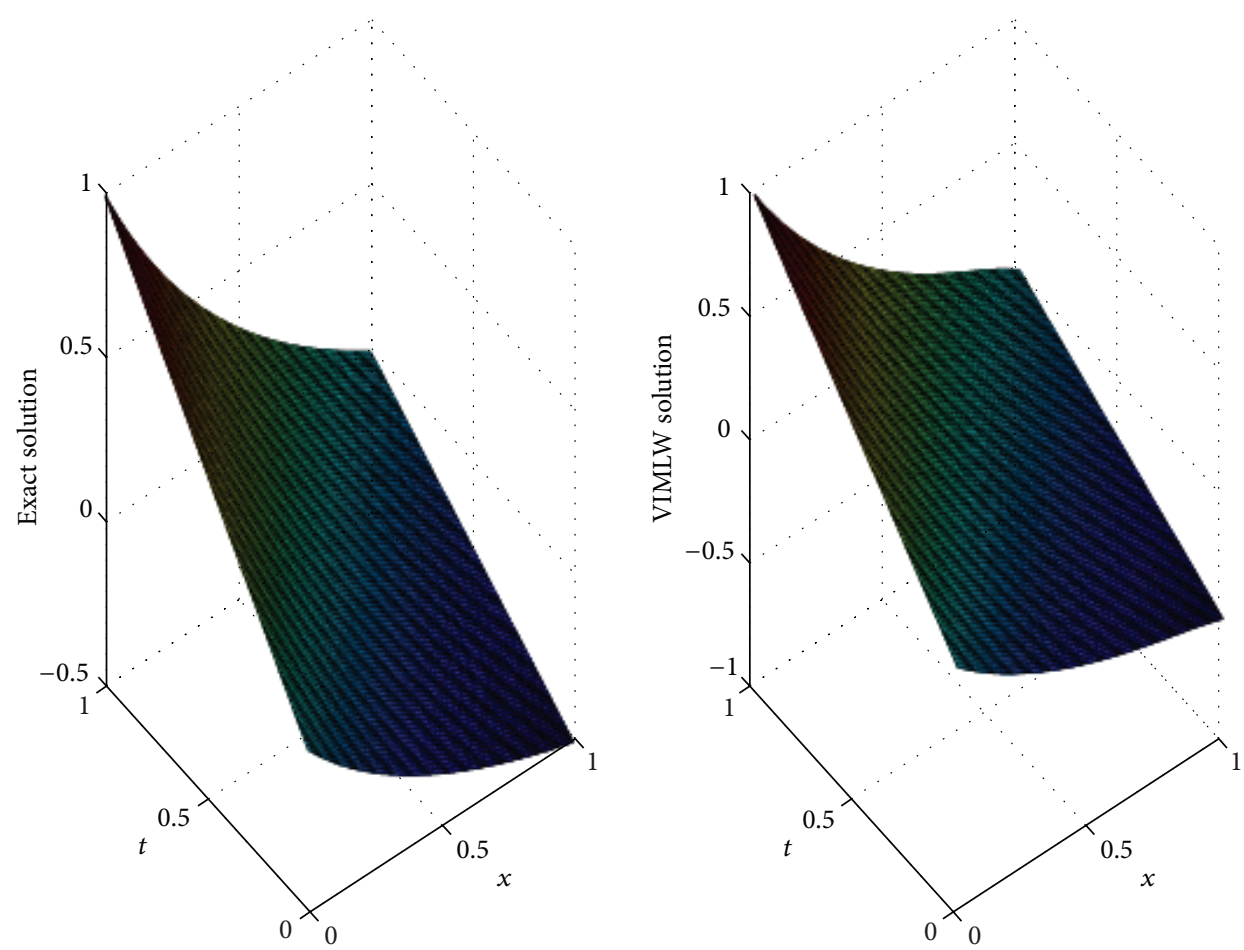

FIGURE 4: Exact solution and VIMLW approximate solution of Example 7.

with VIM. It indicates that the approximate solution is quite close to the exact one.

Example 5. Consider the following equation [39]:

$$
u_{t}+\frac{1}{2} u u_{x x}=u_{x x x}, \quad-\infty<x<\infty, t>0
$$

with the initial conditions $u(x, 0)=x^{2}$, and the exact solution is $u(x, t)=\left(x^{2} /(1+t)\right)$. have

By assuming $u_{k}(x, t)=\Psi^{T}(x) C_{k} \Psi(t)$ and from (54), we

$$
\begin{gathered}
L_{k}\left[u_{k}\right]=\frac{\partial u_{k}}{\partial t}-\frac{\partial^{3} u_{k}}{\partial x^{3}}=\Psi^{T}(x) L_{k} \Psi(t), \\
N_{k}\left[u_{k}\right]=\frac{u_{k}}{2} \frac{\partial^{2} u_{k}}{\partial x^{2}}=\Psi^{T}(x) N_{k} \Psi(t),
\end{gathered}
$$

where $L_{k}=\left(D^{T}\right)^{3} C_{k} D, N_{k}=\left((1 / 2)\left(D^{T}\right)^{2} C_{k}\right) \otimes C_{k}$.

We employ the methods presented in this paper to solve (54) with $M=16$ and $k=1$. The numerical results are presented in Table 2 and shown in Figure 2. It is to be noted that only the fifth-order terms are used in evaluating the approximate solutions. The results obtained using the VIMLW are in good agreement with the results of VIM.

Example 6. We consider the following equation [40]:

$$
\begin{array}{r}
u_{t}(x, t)+u(x, t) u_{x}(x, t)=g(x, t), \\
t>0, x \in R, 0<\alpha \leq 1
\end{array}
$$

with the initial conditions $u(x, 0)=0$ and the exact solution is $u(x, t)=x t$, where $g(x, t)=x+x t^{2}$.

By assuming $u_{k}(x, t)=\Psi^{T}(x) C_{k} \Psi(t), g(x, t)=\Psi^{T}(x)$ $G \Psi(t)$, we have

$$
\begin{gathered}
L_{k}\left[u_{k}\right]=u_{t}(x, t)-g(x, t)=\Psi^{T}(x) L_{k} \Psi(t), \\
N_{k}\left[u_{k}\right]=u_{k} \frac{\partial u_{k}}{\partial x}=\Psi^{T}(x) N_{k} \Psi(t),
\end{gathered}
$$

where $L_{k}=C_{k} D-G, N_{k}=C_{k} \otimes\left(D^{T} C_{k}\right)$.

Table 3 shows the approximate solutions for (56) with $M=16$ and $k=1$ using the VIM and the VIMLW methods and the results are plotted in Figure 3. It is to be noted that only the fourth-order terms of VIM and VIMLW are used in evaluating the approximate solutions in Table 3 . We observe that the approximate solution of (56) with VIMLW gives analogous results to that obtained by VIM, which shows that the approximate solution remains closed form to the exact one.

Example 7. Consider the following Burgers-Poisson (BP) equation of the form [41]:

$$
u_{t}-u_{x x t}+u_{x}+u u_{x}=\left(3 u_{x} u_{x x}+u u_{x x x}\right), \quad-\infty<x<\infty, t>0
$$

with the initial conditions $u(x, 0)=x$, and the exact solution is $u(x, t)=(1+x) /(1+t)-1$. 
TABLE 3: Numerical values when $t=0.25,0.50,0.75$, and 1.0 for (56).

\begin{tabular}{|c|c|c|c|c|c|c|c|c|}
\hline \multirow{2}{*}{$\begin{array}{l}t \\
x\end{array}$} & \multicolumn{4}{|c|}{0.25} & \multicolumn{4}{|c|}{0.50} \\
\hline & 0.25 & 0.50 & 0.75 & 1.00 & 0.25 & 0.50 & 0.75 & 1.00 \\
\hline VIM & 0.06250 & 0.12500 & 0.18750 & 0.25000 & 0.12508 & 0.25015 & 0.37523 & 0.50030 \\
\hline VIMLW & 0.06250 & 0.12500 & 0.18750 & 0.25000 & 0.12508 & 0.25015 & 0.37523 & 0.50030 \\
\hline Exact & 0.06250 & 0.12500 & 0.18750 & 0.25000 & 0.12500 & 0.25000 & 0.37500 & 0.50000 \\
\hline$t$ & \multicolumn{4}{|c|}{0.75} & \multicolumn{4}{|c|}{1.00} \\
\hline$x$ & 0.25 & 0.50 & 0.75 & 1.00 & 0.25 & 0.50 & 0.75 & 1.00 \\
\hline VIM & 0.18882 & 0.37764 & 0.56646 & 0.75527 & 0.25992 & 0.51983 & 0.77975 & 1.03970 \\
\hline VIMLW & 0.18882 & 0.37764 & 0.56646 & 0.75527 & 0.25992 & 0.51983 & 0.77975 & 1.03970 \\
\hline Exact & 0.18750 & 0.37500 & 0.56250 & 0.75000 & 0.25000 & 0.50000 & 0.75000 & 1.00000 \\
\hline
\end{tabular}

TABLE 4: Numerical values when $t=0.25,0.50,0.75$, and 1.0 for (58).

\begin{tabular}{|c|c|c|c|c|c|c|c|c|}
\hline$t$ & \multicolumn{4}{|c|}{0.25} & \multicolumn{4}{|c|}{0.50} \\
\hline$x$ & 0.25 & 0.50 & 0.75 & 1.00 & 0.25 & 0.50 & 0.75 & 1.00 \\
\hline VIM & 0.00009 & 0.20011 & 0.40013 & 0.60015 & -0.16488 & 0.00215 & 0.16917 & 0.33620 \\
\hline VIMLW & 0.00009 & 0.20011 & 0.40013 & 0.59864 & -0.16489 & 0.00215 & 0.16920 & 0.33283 \\
\hline Exact & 0.00000 & 0.20000 & 0.40000 & 0.60000 & -0.16667 & 0.00000 & 0.16667 & 0.33333 \\
\hline$t$ & \multicolumn{4}{|c|}{0.75} & \multicolumn{4}{|c|}{1.00} \\
\hline$x$ & 0.25 & 0.50 & 0.75 & 1.00 & 0.25 & 0.50 & 0.75 & 1.00 \\
\hline VIM & -0.27697 & -0.13237 & 0.01224 & 0.15694 & -0.35057 & -0.22068 & -0.09079 & 0.03909 \\
\hline VIMLW & -0.27701 & -0.13237 & 0.01228 & 0.15213 & -0.35070 & -0.22068 & -0.09078 & 0.03422 \\
\hline Exact & -0.28571 & -0.14286 & 0.00000 & 0.14286 & -0.37500 & -0.25000 & -0.12500 & 0.00000 \\
\hline
\end{tabular}

By assuming $u_{k}(x, t)=\Psi^{T}(x) C_{k} \Psi(t)$, we have

$$
L\left[u_{k}\right]=\frac{\partial u_{k}}{\partial t}-\frac{\partial^{3} u_{k}}{\partial x^{2} \partial t}+\frac{\partial u_{k}}{\partial x}=\Psi^{T}(x) L_{k} \Psi(t),
$$

where $L_{k}=C_{k} D-\left(D^{T}\right)^{2} C_{k} D+D^{T} C_{k}$.

And

$$
N\left[u_{k}\right]=u_{k} \frac{\partial u_{k}}{\partial x}-3 \frac{\partial u_{k}}{\partial x} \frac{\partial^{2} u_{k}}{\partial x^{2}}-u_{k} \frac{\partial^{3} u_{k}}{\partial x^{3}}=\Psi^{T}(x) N_{k} \Psi(t),
$$

where $N_{k}=C_{k} \oplus\left(D^{T} C_{k}\right)-3\left(D^{T} C_{k}\right) \oplus\left[\left(D^{T}\right)^{2} C_{k}\right]-C_{k} \oplus$ $\left[\left(D^{T}\right)^{3} C_{k}\right]$.

Table 4 shows the approximate solutions to (58) with $M=16$ and $k=1$ with VIM and VIMLW, and Figure 4 presents the Exact solution and VIMLW approximate solution of Example 7. Only the fourth-order terms are used in evaluating the approximate solutions in Table 4. From Table 4 and Figure 4 the approximate solution of the given Example 7 by using VIMLW is in good agreement with the results of VIM and it clearly appears that the approximate solution remains closed form to exact solution.

\section{Conclusion}

A new modification of variational iteration method using Legendre wavelets is proposed and employed to solve a number of nonlinear partial differential equations. The proposed method can give approximations of higher accuracy and closed form solutions if existed. There are four important points to make here. First, unlike the VIM, the VIMLW can easily overcome the difficulty arising in the evaluation integration and the derivative of nonlinear terms and does not need symbolic computation. Second, by using the properties of BPFs, operational matrices of product of Legendre wavelets are derived and utilized to deal with nonlinear terms. Third, compared with Legendre wavelets method, the VIMLW only needs a few iterations instead of solving a system of nonlinear algebraic equations. Fourth and most important, VIMLW is computer oriented and can use existing fast algorithms to reduce the computation cost.

\section{Acknowledgments}

This work is supported by the National Natural Science Foundation of China (Grant no. 41105063). The authors are very grateful to the reviewers for carefully reading the paper and for thier comments and suggestions which have improved the paper.

\section{References}

[1] G. Adomian, Solving Frontier Problems of Physics: The Decomposition Method, vol. 60 of Fundamental Theories of Physics, Kluwer Academic Publishers, Dordrecht, The Netherlands, 1994.

[2] G. Adomian, "A review of the decomposition method in applied mathematics," Journal of Mathematical Analysis and Applications, vol. 135, no. 2, pp. 501-544, 1988. 
[3] G. Adomian, "Solutions of nonlinear P. D. E," Applied Mathematics Letters, vol. 11, no. 3, pp. 121-123, 1998.

[4] Q. Esmaili, A. Ramiar, E. Alizadeh, and D. D. Ganji, "An approximation of the analytical solution of the Jeffery-Hamel flow by decomposition method," Physics Letters A, vol. 372, no. 19, pp. 3434-3439, 2008.

[5] A.-M. Wazwaz, "A new algorithm for calculating Adomian polynomials for nonlinear operators," Applied Mathematics and Computation, vol. 111, no. 1, pp. 53-69, 2000.

[6] S. Momani and Z. Odibat, "Analytical solution of a timefractional Navier-Stokes equation by Adomian decomposition method," Applied Mathematics and Computation, vol. 177, no. 2, pp. 488-494, 2006.

[7] J.-H. He, "Homotopy perturbation technique," Computer Methods in Applied Mechanics and Engineering, vol. 178, no. 3-4, pp. 257-262, 1999.

[8] J.-H. He, "A coupling method of a homotopy technique and a perturbation technique for non-linear problems," International Journal of Non-Linear Mechanics, vol. 35, no. 1, pp. 37-43, 2000.

[9] J.-H. He, "The homotopy perturbation method nonlinear oscillators with discontinuities," Applied Mathematics and Computation, vol. 151, no. 1, pp. 287-292, 2004.

[10] S. T. Mohyud-Din and M. A. Noor, "Homotopy perturbation method for solving fourth-order boundary value problems," Mathematical Problems in Engineering, vol. 2007, Article ID 98602, 15 pages, 2007.

[11] S. T. Mohyud-Din and M. A. Noor, "Homotopy perturbation method for solving partial differential equations," Zeitschrift für Naturforschung A, vol. 64, no. 3-4, pp. 157-170, 2009.

[12] S. T. Mohyud-Din and M. A. Noor, "Homotopy perturbation method and Padé approximants for solving Flierl-Petviashivili equation," Applications and Applied Mathematics, vol. 3, no. 2, pp. 224-234, 2008

[13] S. J. Liao, "An approximate solution technique not depending on small parameters: a special example," International Journal of Non-Linear Mechanics, vol. 30, no. 3, pp. 371-380, 1995.

[14] S. J. Liao, "Boundary element method for general nonlinear differential operators," Engineering Analysis with Boundary Elements, vol. 20, no. 2, pp. 91-99, 1997.

[15] J. H. He, "Variational iteration method-a kind of non-linear analytical technique: some examples," International Journal of Non-Linear Mechanics, vol. 34, no. 4, pp. 699-708, 1999.

[16] J.-H. He, "Variational iteration method for autonomous ordinary differential systems," Applied Mathematics and Computation, vol. 114, no. 2-3, pp. 115-123, 2000.

[17] J.-H. He, "Variational principles for some nonlinear partial differential equations with variable coefficients," Chaos, Solitons \& Fractals, vol. 19, no. 4, pp. 847-851, 2004.

[18] J.-H. He and X.-H. Wu, "Construction of solitary solution and compacton-like solution by variational iteration method," Chaos, Solitons \& Fractals, vol. 29, no. 1, pp. 108-113, 2006.

[19] J.-H. He and X.-H. Wu, "Variational iteration method: new development and applications," Computers \& Mathematics with Applications, vol. 54, no. 7-8, pp. 881-894, 2007.

[20] J.-H. He, "Variational iteration method-some recent results and new interpretations," Journal of Computational and Applied Mathematics, vol. 207, no. 1, pp. 3-17, 2007.

[21] J. H. He, G. C. Wu, and F. Austin, "The variational iterational method which should be follow," Nonlinear Science Letters A, vol. 1, no. 1, pp. 1-30, 2010.
[22] J.-H. He, "Some asymptotic methods for strongly nonlinear equations," International Journal of Modern Physics B, vol. 20, no. 10, pp. 1141-1199, 2006.

[23] J. H. He, "Asymptotic methods for solitary solutions and compactons," Abstract and Applied Analysis, vol. 2012, Article ID 916793, 130 pages, 2012.

[24] G. Hariharan, K. Kannan, and K. R. Sharma, "Haar wavelet method for solving Fisher's equation," Applied Mathematics and Computation, vol. 211, no. 2, pp. 284-292, 2009.

[25] Ü. Lepik, "Numerical solution of evolution equations by the Haar wavelet method," Applied Mathematics and Computation, vol. 185, no. 1, pp. 695-704, 2007.

[26] S. G. Venkatesh, S. K. Ayyaswamy, and S. Raja Balachandar, "The Legendre wavelet method for solving initial value problems of Bratu-type," Computers \& Mathematics with Applications, vol. 63, no. 8, pp. 1287-1295, 2012.

[27] S. A. Yousefi, "Legendre wavelets method for solving differential equations of Lane-Emden type," Applied Mathematics and Computation, vol. 181, no. 2, pp. 1417-1422, 2006.

[28] R. K. Pandey, N. Kumar, A. Bhardwaj, and G. Dutta, "Solution of Lane-Emden type equations using Legendre operational matrix of differentiation," Applied Mathematics and Computation, vol. 218, no. 14, pp. 7629-7637, 2012.

[29] F. Yin, J. Song, F. Lu, and H. Leng, "A coupled method of Laplace transform and legendre wavelets for Lane-Emden-type differential equations," Journal of Applied Mathematics, vol. 2012, Article ID 163821, 16 pages, 2012.

[30] L. M. B. Assas, "Variational iteration method for solving coupled-KdV equations," Chaos, Solitons and Fractals, vol. 38, no. 4, pp. 1225-1228, 2008.

[31] Z. M. Odibat, "Construction of solitary solutions for nonlinear dispersive equations by variational iteration method," Physics Letters A, vol. 372, no. 22, pp. 4045-4052, 2008.

[32] E. M. Abulwafa, M. A. Abdou, and A. A. Mahmoud, "The solution of nonlinear coagulation problem with mass loss," Chaos, Solitons \& Fractals, vol. 29, no. 2, pp. 313-330, 2006.

[33] E. M. Abulwafa, M. A. Abdou, and A. A. Mahmoud, "Nonlinear fluid flows in pipe-like domain problem using variationaliteration method," Chaos, Solitons \& Fractals, vol. 32, no. 4, pp. 1384-1397, 2007.

[34] N. Bildik and A. Konuralp, "The use of variational iteration method, differential transform method and adomian decomposition method for solving different types of nonlinear partial differential equations," International Journal of Nonlinear Sciences and Numerical Simulation, vol. 7, no. 1, pp. 65-70, 2006.

[35] S. Momani and S. Abuasad, "Application of He's variational iteration method to Helmholtz equation," Chaos, Solitons \& Fractals, vol. 27, no. 5, pp. 1119-1123, 2006.

[36] S. Momani and Z. Odibat, "Numerical comparison of methods for solving linear differential equations of fractional order," Chaos, Solitons \& Fractals, vol. 31, no. 5, pp. 1248-1255, 2007.

[37] S. T. Mohyud-Din, M. A. Noor, K. I. Noor, and M. M. Hosseini, "Solution of singular equation by He's variational iteration method," International Journal of Nonlinear Sciences and Numerical Simulation, vol. 11, no. 2, pp. 81-86, 2010.

[38] N. H. Sweilam and M. M. Khader, "Variational iteration method for one dimensional nonlinear thermoelasticity," Chaos, Solitons \& Fractals, vol. 32, no. 1, pp. 145-149, 2007.

[39] D. D. Ganji, H. Tari, and M. Bakhshi Jooybari, "Variational iteration method and homotopy perturbation method for nonlinear evolution equations," Computers \& Mathematics with Applications, vol. 54, no. 7-8, pp. 1018-1027, 2007. 
[40] Z. Odibat and S. Momani, "Numerical methods for nonlinear partial differential equations of fractional order," Applied Mathematical Modelling, vol. 32, no. 1, pp. 28-39, 2008.

[41] E. Hizel and S. K. Küçükarslan, "A numerical analysis of the Burgers-Poisson (BP) equation using variational iteration method," in Proceedings of the 3rd WSEAS International Conference on Applied and Theoretical Mechanics, Tenerife, Spain, December 2007.

[42] S. T. Mohyud-Din and M. A. Noor, "Modified variational iteration method for solving Fisher's equations," Journal of Applied Mathematics and Computing, vol. 31, no. 1-2, pp. 295308, 2009.

[43] M. A. Noor and S. T. Mohyud-Din, "Variational iteration method for solving higher-order nonlinear boundary value problems using He's polynomials," International Journal of Nonlinear Sciences and Numerical Simulation, vol. 9, no. 2, pp. 141-156, 2008.

[44] M. A. Noor and S. T. Mohyud-Din, "Modified variational iteration method for heat and wave-like equations," Acta Applicandae Mathematicae, vol. 104, no. 3, pp. 257-269, 2008.

[45] M. A. Noor and S. T. Mohyud-Din, "Modified variational iteration method for solving Helmholtz equations," Computational Mathematics and Modeling, vol. 20, no. 1, pp. 40-50, 2009.

[46] M. A. Noor and S. T. Mohyud-Din, "Variational iteration method for fifth-order boundary value problems using He's polynomials," Mathematical Problems in Engineering, vol. 2008, Article ID 954794, 12 pages, 2008.

[47] M. A. Noor and S. T. Mohyud-Din, "Modified variational iteration method for solving fourth-order boundary value problems," Journal of Applied Mathematics and Computing, vol. 29, no. 1-2, pp. 81-94, 2009.

[48] M. A. Noor and S. T. Mohyud-Din, "Modified variational iteration method for Goursat and Laplace problems," World Applied Sciences Journal, vol. 4, no. 4, pp. 487-498, 2008.

[49] S. Abbasbandy, "A new application of He's variational iteration method for quadratic Riccati differential equation by using Adomian's polynomials," Journal of Computational and Applied Mathematics, vol. 207, no. 1, pp. 59-63, 2007.

[50] S. Abbasbandy, "Numerical solution of non-linear KleinGordon equations by variational iteration method," International Journal for Numerical Methods in Engineering, vol. 70, no. 7, pp. 876-881, 2007.

[51] S. T. Mohyud-Din and M. A. Noor, "Solving Schrödinger equations by modified variational iteration method," World Applied Sciences Journal, vol. 5, no. 3, pp. 352-357, 2008.

[52] S. T. Mohyud-Din, M. A. Noor, and K. I. Noor, "Modified variational iteration method for solving Sine Gordon equations," World Applied Sciences Journal, vol. 5, no. 3, pp. 352-357, 2008.

[53] M. A. Noor and S. T. Mohyud-Din, "Solution of singular and nonsingular initial and boundary value problems by modified variational iteration method," Mathematical Problems in Engineering, vol. 2008, Article ID 917407, 23 pages, 2008.

[54] M. A. Noor and S. T. Mohyud-Din, "Variational iteration decomposition method for solving eighth-order boundary value problems," Differential Equations and Nonlinear Mechanics, vol. 2007, Article ID 19529, 16 pages, 2007.

[55] M. M. Hosseini, "Adomian decomposition method with Chebyshev polynomials," Applied Mathematics and Computation, vol. 175, no. 2, pp. 1685-1693, 2006.

[56] W. C. Tien and C. K. Chen, "Adomian decomposition method by Legendre polynomials," Chaos, Solitons and Fractals, vol. 39, no. 5, pp. 2093-2101, 2009.
[57] Z. Odibat, "On Legendre polynomial approximation with the VIM or HAM for numerical treatment of nonlinear fractional differential equations," Journal of Computational and Applied Mathematics, vol. 235, no. 9, pp. 2956-2968, 2011.

[58] M. Razzaghi and S. Yousefi, "The Legendre wavelets operational matrix of integration," International Journal of Systems Science, vol. 32, no. 4, pp. 495-502, 2001.

[59] F. Mohammadi and M. M. Hosseini, "A new Legendre wavelet operational matrix of derivative and its applications in solving the singular ordinary differential equations," Journal of the Franklin Institute, vol. 348, no. 8, pp. 1787-1796, 2011. 


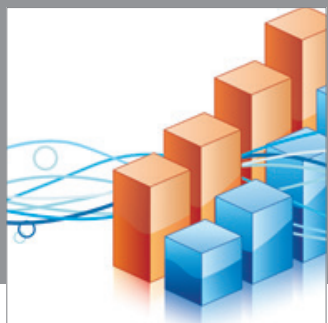

Advances in

Operations Research

mansans

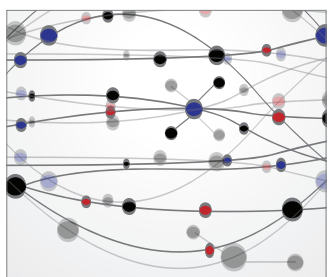

The Scientific World Journal
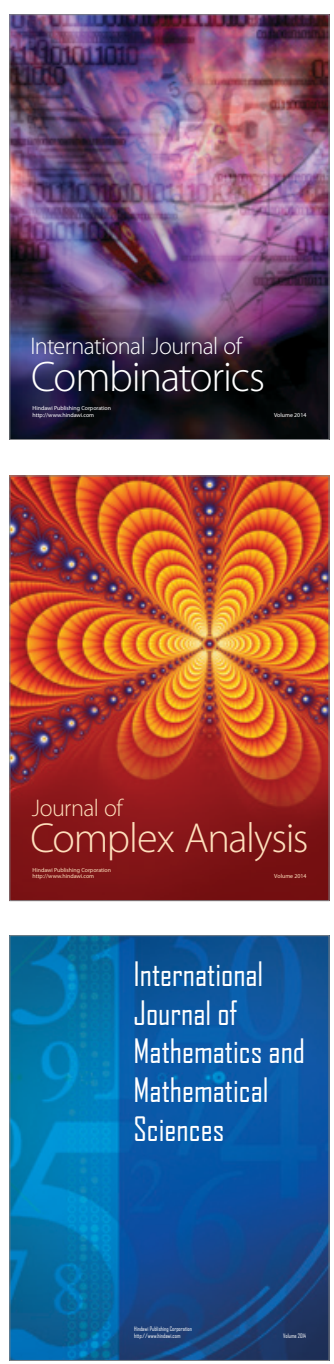
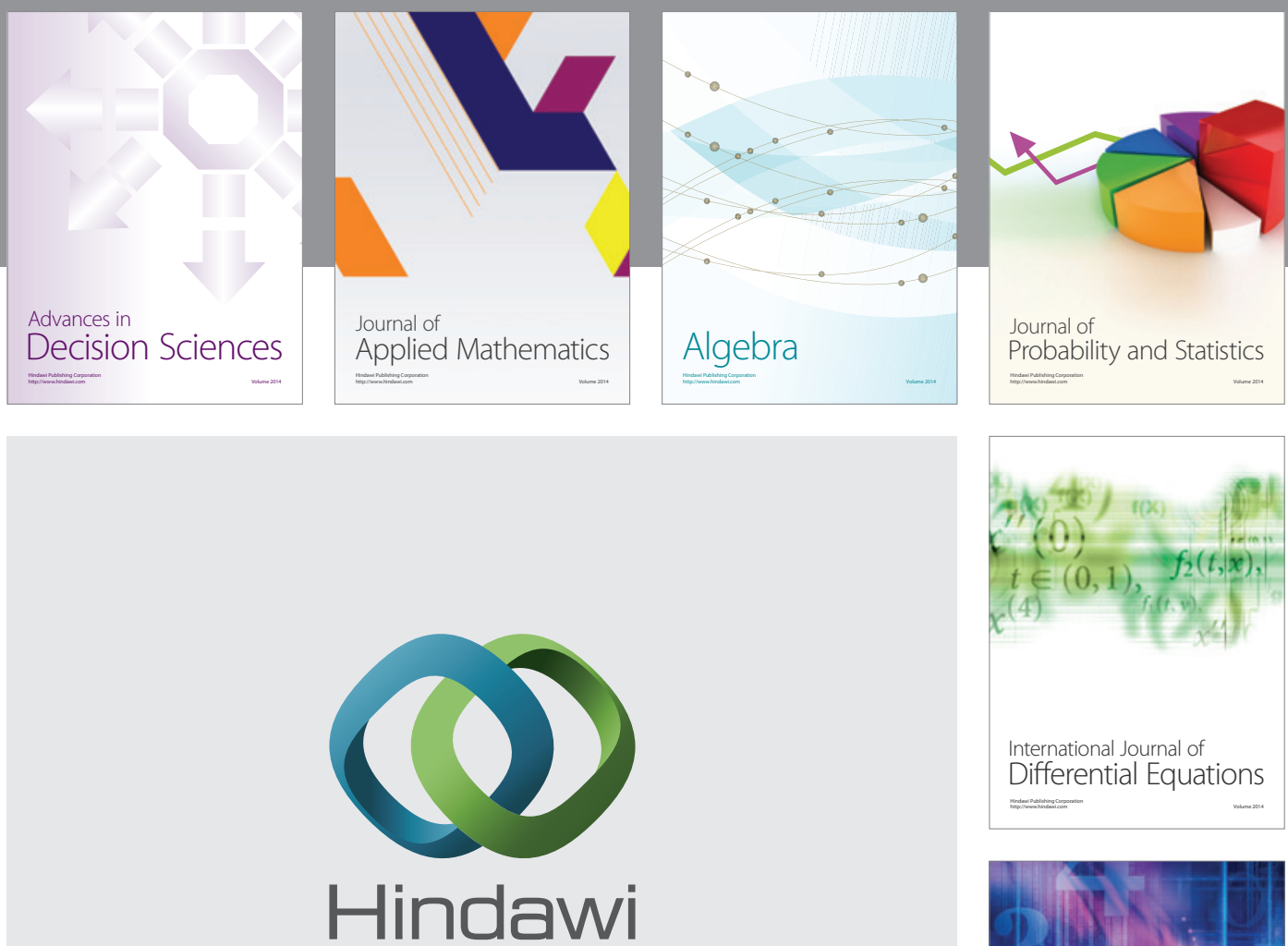

Submit your manuscripts at http://www.hindawi.com
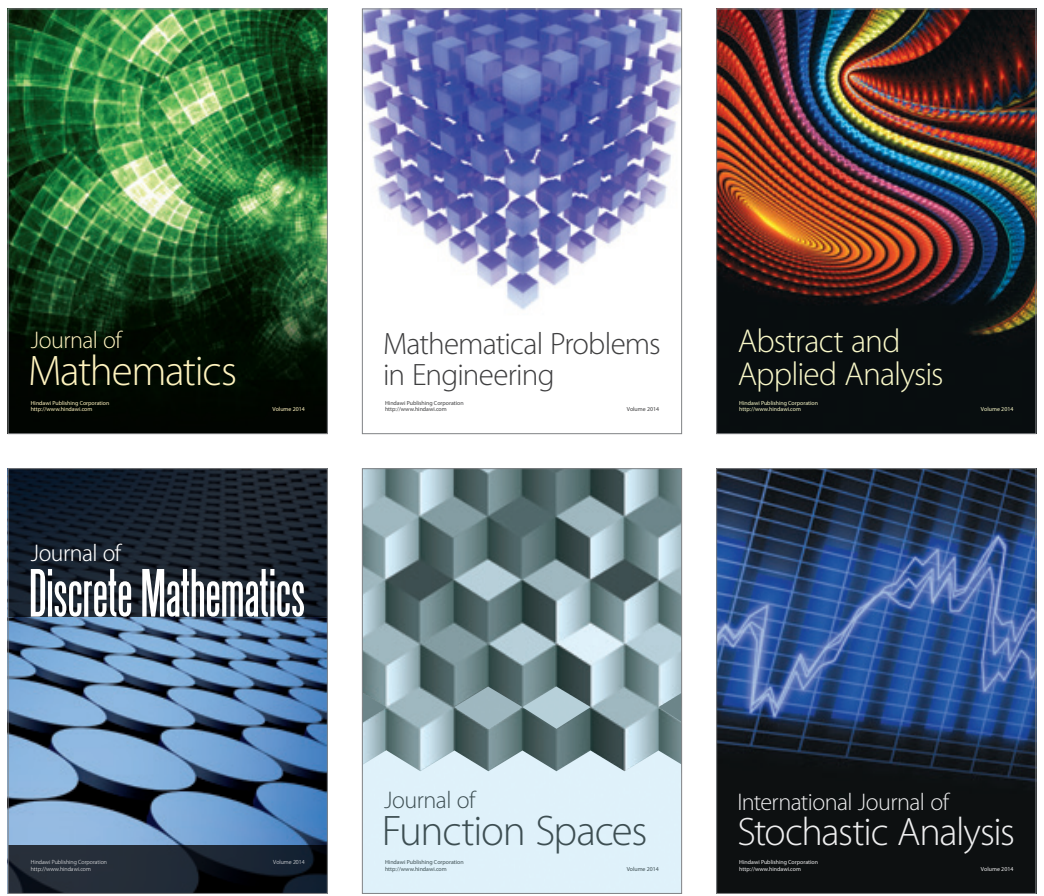

Journal of

Function Spaces

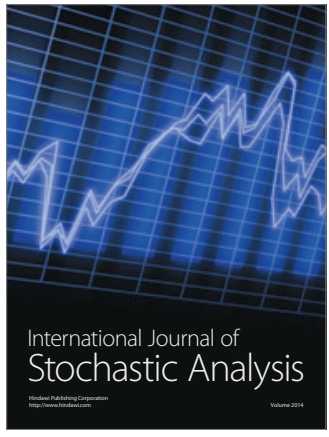

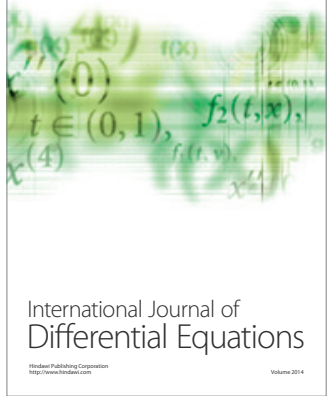
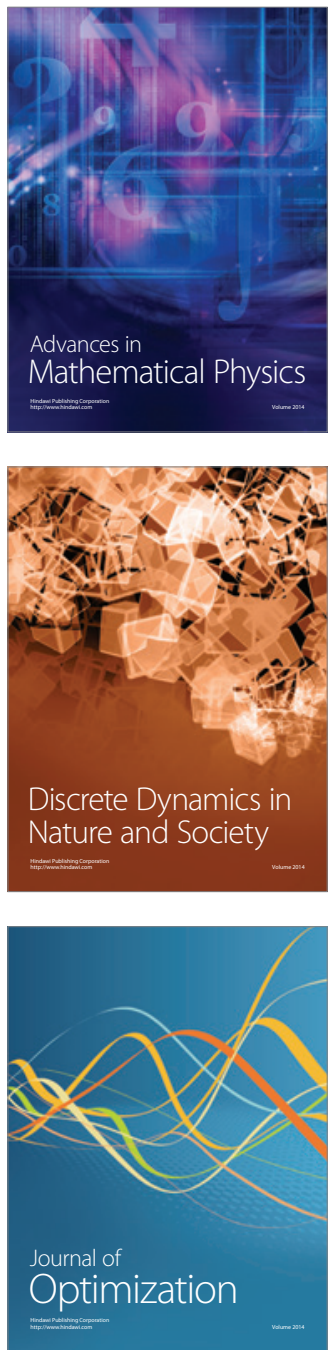